\title{
Series Expansions for Analytic Systems Linear in the Controls
}

\author{
Francesco Bullo \\ Coordinated Science Laboratory and General Engineering Department \\ University of Illinois at Urbana-Champaign \\ 1308 W. Main St, Urbana, IL 61801, USA \\ Tel: (217) 333-0656, Fax: (217) 244-1653 \\ Email: bullo@uiuc.edu, Url: http://motion.csl.uiuc.edu
}

\begin{abstract}
This paper presents a series expansion for the evolution of nonlinear systems which are analytic in the state and linear in the controls. An explicit recursive expression is obtained assuming that the input vector fields are constant. Additional simplifications take place in the analysis of systems described by second order polynomial vector fields. Sufficient conditions are derived to guarantee uniform convergence over the finite and infinite time horizon. The treatment relies only on elementary notions on analytic functions, number theory and operator norms.
\end{abstract}

\section{Introduction}

This papers studies series expansions for the evolution of a class of nonlinear control system. Series expansions play a key rôle in the study of sufficient and necessary conditions for local nonlinear controllability; see Sussmann [20] and Kawski [8], and in other areas such as geometric integration; see McLachlan et al. [16], and realization theory; see Isidori [7, Section 3.4 and 3.5]. Additionally, series expansions are an enabling tool in trajectory generation and optimization problems, e.g., see Lafferriere and Sussmann [13], Leonard and Krishnaprasad [14] and the author's work [4]. In these algorithms, simple computable expressions and small truncation errors are desirable.

Volterra series and other types of expansions have been the subject of attention of many researchers. Some early work includes Brockett [1], Gilbert [6], Lesiak and Krener [15]. Later Fliess [5] provided a comprehensive treatment to what is now known as the Chen-Fliess series. Motivated by controllability and normal form theory, Sussmann and Kawski [9, 19] obtained increasingly sophisticated versions of the Chen-Fliess series. On a related line of research, the textbook Rugh [17] focuses on the input/output representation of nonlin- ear systems via series expansions: this is sometimes referred to as the Volterra/Wiener approach to nonlinear control. Finally, the author presented in $[2,3]$ a series expansion for mechanical systems specially tailored to the the homogeneous structure of such systems.

This paper presents novel series expansions for nonlinear control systems described by analytic drift vector fields and constant input fields. We call such systems "linear in the controls." We obtain recursive expressions for the general case, as well as we illustrate how they further simplify when dealing with vector fields with polynomial components. The presentation and derivation rely only on elementary tools and the final expansions appear in a format similar but not identical to the classic Volterra format.

Asymptotic bounds on the truncation error and convergence properties for the series are fully characterized. The series expansion converges uniformly over all time provided the linearized system is stable and the input norm is bounded by a computable constant. Alternatively, for an arbitrary input, the series is guaranteed to converge over a specific finite interval of time, where the time lower bound is computable. These results are in agreement with the classic limitation of perturbation and averaging methods in dynamical systems, e.g., see Khalil [10, Section 8.2] and Sanders and Verhulst [18, page 71]. We refer the reader to a later publication for a more thorough comparison of this paper with the literature on Volterra series.

\section{Preliminaries}

Some elementary number theory: We refer to [11] for some basic introduction into the subject of generating function. We quickly review some notation.

Let $\mathbb{N}$ be the set of positive integer numbers, $\mathbb{R}$ the set of real numbers and $\mathbb{C}$ the set of complex numbers. Let $k \in \mathbb{N}$, and let $P(k)$ be the set of or- 
dered partitions of $k$. For example, $P(3)$ is the collection $\{\{3\},\{2,1\},\{1,2\},\{1,1,1\}\}$. It is easy to see that $P(k)$ contains $2^{k-1}$ elements. Let $P(i, j)$ be the set of ordered sequences of $j$ integers that sums up to $i$, and let $P(k)-\{k\}$ be the set $P(k)$ minus the element $\{k\}$.

The initial value problem and Taylor expansions: Let $x$ take value in $\mathbb{R}^{n}$ and let $t$ belong to an interval $I$ : the finite time case, i.e., $I=[0, T]$, as well as the infinite horizon case, i.e., $I=[0, \infty)$, are of interest. Consider the initial value problem

$$
\begin{aligned}
\dot{x}(t) & =f(x(t))+g(t) \\
x(0) & =0,
\end{aligned}
$$

where components $\left\{f_{i}, i=1, \ldots, n\right\}$ of $f$ are analytic functions in a neighborhood of the origin $0 \in \mathbb{R}^{n}$. Let $g_{i}$ be the $i$ th component of $g$ and assume it to be integrable over the interval $I$. The initial value problem (1) is thought of as a control system by setting $g(t)=B u(t)$; we refer to this system as with additive controls as the inputs appear independently of $x$.

Let $f(0)=0$, and for $i=1, \ldots, n$, develop the functions $f_{i}$ in a Taylor expansion about the origin via

$$
\begin{aligned}
f_{i}(x)=\sum_{\substack{m=1 \\
j_{1}+\cdots+j_{n}=m \\
j_{1}, \ldots, j_{n}>0}} \frac{1}{j_{1} ! \cdots j_{n} !} \\
\\
\quad\left(\frac{\partial^{m}}{\partial x_{1}^{j_{1}} \ldots \partial x_{n}^{j_{n}}} f_{i}(0)\right) x_{1}^{j_{1}} \cdots x_{n}^{j_{n}} .
\end{aligned}
$$

Equivalently, let

$$
f_{i}(x)=\sum_{m=1}^{+\infty} f_{i}^{[m]}(x, \ldots, x),
$$

where for all $m \in \mathbb{N}$ the tensors $f_{i}^{[m]}: \underbrace{\mathbb{R}^{n} \times \ldots \times \mathbb{R}^{n}}_{m} \rightarrow$ $\mathbb{R}^{n}$ are computed according to

$$
\begin{aligned}
& m ! f_{i}^{[m]}\left(y_{1}, \ldots, y_{m}\right) \\
& =\sum_{k_{1}, \ldots, k_{m}=1}^{n}\left(\frac{\partial^{m}}{\partial x_{k_{1}} \ldots \partial x_{k_{m}}} f_{i}(0)\right)\left(y_{1}\right)_{k_{1}} \cdots\left(y_{m}\right)_{k_{m}} \\
& \quad=m ! \sum_{k_{1}, \ldots, k_{m}=1}^{n}\left(f_{i}^{[m]}\right)^{k_{1} \ldots k_{m}}\left(y_{1}\right)_{k_{1}} \cdots\left(y_{m}\right)_{k_{m}},
\end{aligned}
$$

and the vectors $y_{1}, \ldots, y_{m}$ belong to $\mathbb{R}^{n}$. These definitions and results are readily repeated in vector format for the vector field $f$ simply by neglecting the subscript $i$. It will be convenient to adopt the notation $A x=f^{[1]}(x)$.

Remark 2.1 The sequence of tensors $\left\{f^{[m]}, m \in \mathbb{N}\right\}$ uniquely determines the Lie algebraic structure at the origin of the control system in equation (1). For example, one can see that

$$
\left.\frac{1}{2}\left[g\left(t_{2}\right),\left[g\left(t_{1}\right), f(x)\right]\right]\right|_{x=0}=f^{[2]}\left(g\left(t_{1}\right), g\left(t_{2}\right)\right) .
$$

Some operator norms and their estimates: In defining mapping and norms we follow the notation in [10, Chapter 6]. Consider the normed linear space $\mathcal{L}_{\infty}^{n}$ of piecewise continuous, uniformly bounded functions over the interval $I$

$$
\begin{aligned}
x: I \subset \mathbb{R}_{+} & \rightarrow \mathbb{R}^{n} \\
t & \mapsto x(t),
\end{aligned}
$$

with norm

$$
\|x\|_{\mathcal{L}_{\infty}}=\sup _{t \in I}\|x(t)\|_{\infty}=\sup _{t \in I} \max _{i=1, \ldots, n}\left|x_{i}(t)\right|<\infty .
$$

Assume the matrix $A$ is Hurwitz or that the interval $I$ is finite, and let $H_{A}$ be the mapping

$$
\begin{aligned}
H_{A}: \mathcal{L}_{\infty}^{n} & \rightarrow \mathcal{L}_{\infty}^{n} \\
x(t) & \mapsto \int_{0}^{t} \mathrm{e}^{A(t-\tau)} x(\tau) d \tau .
\end{aligned}
$$

The $\mathcal{L}_{\infty}^{n}$ iinduced norm for $H_{A}$ is

$$
\left\|H_{A}\right\|_{\mathcal{L}_{\infty}}=\left\|\mathrm{e}^{A t}\right\|_{\mathcal{L}_{1}}=\max _{i=1, \ldots, n} \sum_{j=1}^{n} \int_{t \in I}\left|\left(\mathrm{e}^{A t}\right)_{i j}\right| d t .
$$

Next, we consider the vector field $f$ and its derived tensors $f^{[m]}$. For simplicity and for later use we start by considering the 2 -tensor $f^{[2]}: \mathcal{L}_{\infty}^{n} \times \mathcal{L}_{\infty}^{n} \rightarrow \mathcal{L}_{\infty}^{n}$ defined via

$$
(x(t), y(t)) \mapsto f^{[2]}(x(t), y(t)) .
$$

and defining its induced norm $\left\|f^{[2]}\right\|_{\mathcal{L}_{\infty}}$ via

$$
\left\|f^{[2]}\right\|_{\mathcal{L}_{\infty}} \triangleq \max _{\substack{\left\|y_{1}\right\|_{\mathcal{L}_{\infty}=1} \\\left\|y_{2}\right\|_{\mathcal{L}_{\infty}=1}}}\left\|f^{[2]}\left(y_{1}, y_{2}\right)\right\|_{\mathcal{L}_{\infty}} .
$$

More generally, we examine the $m$-tensor $f^{[m]}$ and define its induced norm via

$$
\begin{aligned}
& \left\|f^{[m]}\right\|_{\mathcal{L}_{\infty}} \triangleq \max _{\substack{\left\|y_{j}\right\|_{\mathcal{L}_{\infty}=1}=1 \\
j=1, \ldots, m}}\left\|f^{[m]}\left(y_{1}, \ldots, y_{j}\right)\right\|_{\mathcal{L}_{\infty}} \\
& \quad \leq \max _{i=1, \ldots, n} \sum_{k_{1}, \ldots, k_{m}=1}^{n} \frac{1}{m !}\left|\frac{\partial^{m}}{\partial x_{k_{1}} \ldots \partial x_{k_{m}}} f_{i}(0)\right| .
\end{aligned}
$$

Note that the estimates on the norm of the mappings are only upper bounds whenever $m \geq 2$.

An upper bound on the operator norm $\left\|f^{[m]}\right\|_{\mathcal{L}_{\infty}}$ is provided by the Cauchy estimates for the Taylor series 
coefficient of an analytic function, see [12, Section 2.3]. Since we assumed the vector field $f$ analytic about the origin, there exists a $\rho \in \mathbb{R}_{+}$such that $f$ is analytic over the domain

$$
D_{\rho}=\left\{\left(z_{1}, \ldots, z_{n}\right) \in \mathbb{C}^{n}|| z_{i} \mid<\rho\right\} .
$$

We let $\|f\|_{\rho}$ denote the maximum value attained by the magnitude of all components of $f$ over $D_{\rho}$. The classic result states that for any collection of nonnegative indices $j_{1}+\cdots+j_{n}=m$,

$$
\left|\frac{\partial^{m}}{\partial x_{1}^{j_{1}} \ldots \partial x_{n}^{j_{n}}} f_{i}(0)\right| \leq \frac{j_{1} ! \cdots j_{n} !}{\rho^{m}}\|f\|_{\rho} .
$$

Conservatively bounding the right hand side by $\left(m ! / \rho^{m}\right)\|f\|_{\rho}$, we have

$$
\begin{aligned}
\left\|f^{[m]}\right\|_{\mathcal{L}_{\infty}} & \leq \sum_{k_{1}, \ldots, k_{m}=1}^{n} \frac{1}{m !} \frac{j_{1} ! \cdots j_{n} !}{\rho^{m}}\|f\|_{\rho} \\
& \leq \frac{1}{\rho^{m}}\|f\|_{\rho} \sum_{k_{1}, \ldots, k_{m}=1}^{n} 1=\left(\frac{n}{\rho}\right)^{m}\|f\|_{\rho} .
\end{aligned}
$$

Finally, given any scalar function $h$ of a scalar variable $\eta$, we let $\operatorname{Remainder}_{M}(h)(\eta)$ be its Taylor remainder of order $M$.

\section{A series expansion}

Let $\epsilon$ be a positive constant and consider the initial value problem

$$
\begin{aligned}
& \dot{x}(t, \epsilon)=f(x(t, \epsilon))+\epsilon g(t) \\
& x(0, \epsilon)=0,
\end{aligned}
$$

where the solution $x$ is a function of both $t \in I$ and $\epsilon \in \mathbb{R}_{+}$. Following is the main result of the paper.

Proposition 3.1 Consider the initial value problem in equation (2). The solution $x: I \times \mathbb{R}_{+} \mapsto \mathbb{R}^{n}$ satisfies the formal expansion

$$
\begin{aligned}
x(t, \epsilon) & =\sum_{k=1}^{+\infty} \epsilon^{k} x_{k}(t) \\
x_{1}(t) & =\int_{0}^{t} \mathrm{e}^{A(t-\tau)} g(\tau) d \tau \\
x_{k}(t) & =\sum_{\left\{i_{1} \ldots i_{m}\right\} \in P(k)-\{k\}} \int_{0}^{t} \mathrm{e}^{A(t-\tau)} f^{[m]}\left(x_{i_{1}}, \ldots, x_{i_{m}}\right) d \tau,
\end{aligned}
$$

where the argument $\tau$ is dropped for simplicity.

Assume $f$ analytic over the domain $D_{\rho}$. Without loss of generality let $\|g\|_{\mathcal{L}_{\infty}}=\|f\|_{\rho}$, and compute

$$
\beta=\left(\frac{n}{\rho}\right)\left\|\mathrm{e}^{A t}\right\|_{\mathcal{L}_{1}}\|f\|_{\rho} .
$$

If $\beta \epsilon \leq 1+2 \beta-2 \sqrt{\beta+\beta^{2}}$, the series in equation (3) converges absolutely and uniformly in $t \in I$, and for all integers $M$ the truncation error is bounded by

$$
\left\|x-\sum_{k=1}^{M} x_{k}\right\|_{\mathcal{L}_{\infty}} \leq\left(\frac{\rho}{n}\right) \operatorname{Remainder}_{M}\left(h_{\beta}\right)(\beta \epsilon),
$$

where

$$
h_{\beta}(\eta)=\frac{1+\eta-\sqrt{1-2(1+2 \beta) \eta+\eta^{2}}}{2(\beta+1)} .
$$

Proof: The following notation is inspired by the treatment in [10, Section 8.1] on the perturbation method. In computing power series expansions of a generic function $y(\epsilon)$, it will be convenient to use the notation

$$
\left[\epsilon^{k}\right] y(t, \epsilon)
$$

for the coefficient of $\epsilon^{k}$ in $y(t, \epsilon)$; this notation is taken from [11, Section 1.2.9]. For the left side of equation (2) we easily have

$$
\left[\epsilon^{k}\right] \dot{x}(t, \epsilon)=\left[\epsilon^{k}\right] \sum_{j=1}^{+\infty} \epsilon^{j} \dot{x}_{j}(t)=\dot{x}_{k}(t) .
$$

For the right hand side we compute

$$
\begin{gathered}
\left.f(x)\right|_{x=\sum_{j=1}^{+\infty} x_{j}}+\epsilon g(t)=\epsilon g(t)+ \\
\left.\left(f^{[1]}(x)+\ldots+f^{[m]}(x, \ldots, x) \ldots\right)\right|_{x=\epsilon x_{1}+\ldots+\epsilon^{j} x_{j} \ldots}
\end{gathered}
$$

The coefficient of $\epsilon$ is $f^{[1]}\left(x_{1}\right)+g(t)=A x_{1}+g(t)$, and accordingly

$$
\begin{aligned}
\dot{x}_{1} & =[\epsilon](f(x)+\epsilon g(t)) \\
& =A x_{1}+g(t) .
\end{aligned}
$$

The definition of $x_{1}$ in the proposition follows from noting that the initial condition of $x_{1}$, as well as for any other $x_{k}$, is zero. We compute the coefficient of $\epsilon^{k}$ as follows:

$$
\begin{aligned}
& {\left[\epsilon^{k}\right] f(x(t, \epsilon))=} \\
& =\left[\epsilon^{k}\right] \sum_{m=1}^{+\infty} f^{[m]}\left(\sum_{i_{1}=1}^{+\infty} \epsilon^{i_{1}} x_{i_{1}}, \ldots, \sum_{i_{m}=1}^{+\infty} \epsilon^{i_{m}} x_{i_{m}}\right) \\
& =\sum_{m=1}^{k}\left[\epsilon^{k}\right] f^{[m]}\left(\sum_{i_{1}=1}^{k} \epsilon^{i_{1}} x_{i_{1}}, \ldots, \sum_{i_{m}=1}^{k} \epsilon^{i_{m}} x_{i_{m}}\right) \\
& =\sum_{m=1}^{k} \sum_{\left\{i_{1} \ldots i_{m}\right\} \in P(k, m)} f^{[m]}\left(x_{i_{1}}, \ldots, x_{i_{m}}\right) \\
& =f^{[1]}\left(x_{k}\right)+\sum_{\left\{i_{1} \ldots i_{m}\right\} \in P(k)-\{k\}} f^{[m]}\left(x_{i_{1}}, \ldots, x_{i_{m}}\right),
\end{aligned}
$$


where $P(k, m)$ is the set of ordered sequences of $m$ integers summing up to $k$. The differential equation for the order $\epsilon^{k}$ term is therefore

$$
\dot{x}_{k}=A x_{k}+\sum_{\left\{i_{1} \ldots i_{m}\right\} \in P(k)-\{k\}} f^{[m]}\left(x_{i_{1}}, \ldots, x_{i_{m} s}\right) .
$$

This proves the recursive definition of $x_{k}$.

In the second part of the proof we seek an upper bound on $\epsilon$ which guarantees that the series in equation (3) converges absolutely and uniformly over $t \in I$. Using the operator norms and bounds discussed in Section 2, we compute

$$
\left\|x_{1}\right\|_{\mathcal{L}_{\infty}} \leq\left\|\mathrm{e}^{A t}\right\|_{\mathcal{L}_{1}}\|g\|_{\mathcal{L}_{\infty}}=\left\|\mathrm{e}^{A t}\right\|_{\mathcal{L}_{1}}\|f\|_{\mathcal{L}_{\infty}},
$$

and

$$
\begin{aligned}
\left\|x_{k}\right\|_{\mathcal{L}_{\infty}} & \leq\left\|\mathrm{e}^{A t}\right\|_{\mathcal{L}_{1}} \\
& \times \sum_{\left\{i_{1} \ldots i_{m}\right\} \in P(k)-\{k\}}\left\|f^{[m]}\right\|_{\mathcal{L}_{\infty}}\left\|x_{i_{1}}\right\|_{\mathcal{L}_{\infty}} \cdots\left\|x_{i_{m}}\right\|_{\mathcal{L}_{\infty}} .
\end{aligned}
$$

The summation in the last equation can be rewritten as

$$
\|f\|_{\rho} \sum_{\left\{i_{1} \ldots i_{m}\right\} \in P(k)-\{k\}}\left(\frac{n}{\rho}\right)^{m}\left\|x_{i_{1}}\right\|_{\mathcal{L}_{\infty}} \cdots\left\|x_{i_{m}}\right\|_{\mathcal{L}_{\infty}} .
$$

Let $\beta=\left(\frac{n}{\rho}\right)\left\|\mathrm{e}^{A t}\right\|_{\mathcal{L}_{1}}\|f\|_{\rho}$, and define the series of positive numbers $a_{1}=1$, and

$$
a_{k}=\beta \sum_{\left\{i_{1} \ldots i_{m}\right\} \in P(k)-\{k\}} a_{i_{1}} \cdots a_{i_{m}}
$$

or equivalently

$$
a_{k}=\frac{\beta}{1+\beta} \sum_{\left\{i_{1} \ldots i_{m}\right\} \in P(k)} a_{i_{1}} \cdots a_{i_{m}} .
$$

By induction one can show that

$$
\left\|x_{k}\right\|_{\mathcal{L}_{\infty}} \leq\left(\frac{\rho}{n}\right) \beta^{k} a_{k} .
$$

To characterize the behavior of the sequence $\left\{a_{k}\right\}$ we resort to the method of generating functions; see [11]. We introduce the function $h(\eta)=\sum_{k=1}^{+\infty} a_{k} \eta^{k}$, and study it as follows:

$$
\begin{aligned}
h(\eta) & =\eta+\frac{\beta}{\beta+1} \sum_{k=2}^{+\infty} \eta^{k} \sum_{\left\{i_{1} \ldots i_{m}\right\} \in P(k)} a_{i_{1}} \cdots a_{i_{m}} \\
& =\eta+\frac{\beta}{\beta+1} \sum_{k=2}^{+\infty} \sum_{\left\{i_{1} \ldots i_{m}\right\} \in P(k)}\left(a_{i_{1}} \eta^{i_{1}}\right) \cdots\left(a_{i_{m}} \eta^{i_{m}}\right) .
\end{aligned}
$$

The summation from $k=2, \ldots,+\infty$ is rewritten as

$$
-\eta+\sum_{k=1}^{+\infty} \sum_{\left\{i_{1} \ldots i_{m}\right\} \in P(k)}\left(a_{i_{1}} \eta^{i_{1}}\right) \cdots\left(a_{i_{m}} \eta^{i_{m}}\right) .
$$

In the spirit of the generating function method one performs the simplification

$$
\begin{aligned}
\sum_{k=1}^{+\infty} & \sum_{\left\{i_{1} \ldots i_{m}\right\} \in P(k)}\left(a_{i_{1}} \eta^{i_{1}}\right) \cdots\left(a_{i_{m}} \eta^{i_{m}}\right) \\
= & \sum_{j=1}^{+\infty}\left(\sum_{i=1}^{+\infty} a_{i} \eta^{i}\right)^{j}=\sum_{j=1}^{+\infty}(h(\eta))^{j}=\frac{h(\eta)}{1-h(\eta)},
\end{aligned}
$$

where the first equality is equivalent to equation (6) and the last equality holds under the assumption $h<1$. This bound will be established a posteriori. The rest is ordinary algebra. We compute $h$ as a function of $\eta$ from the equation

$$
h=\eta+\frac{\beta}{\beta+1}\left(-\eta+\frac{h}{1-h}\right)
$$

to obtain ${ }^{1}$

$$
h(\eta)=\frac{1+\eta-\sqrt{1-2(1+2 \beta) \eta+\eta^{2}}}{2(\beta+1)} .
$$

The function $h$ is defined real for any $0 \leq \eta \leq 1+2 \beta-$ $2 \sqrt{\beta+\beta^{2}}$ and over this domain it attains a maximum value of

$$
\max _{0 \leq \eta \leq 1+2 \beta-2 \sqrt{\beta+\beta^{2}}} h=1-\sqrt{\frac{\beta}{\beta+1}} .
$$

As the parameter $\beta$ increases, the convergence region and the maximum value of $h$ diminishes. The bound on $\eta$ translates into a (conservative) estimate on how large $\epsilon$ can be in order for the series in equation (3) to converge. The bound on $h(\eta)$ translates into an estimate of the corresponding norm of the displacement $\|x(t)\|_{\mathcal{L}_{\infty}}$ over the domain of guaranteed convergence. In any case $h$ is always less than unity, so that the bound $h<1$ is justified a posteriori.

Finally, from the estimate in equation (7) we obtain

$$
\begin{aligned}
\left\|x-\sum_{k=1}^{M} x_{k}\right\|_{\mathcal{L}_{\infty}} & \leq \sum_{k>M} \epsilon^{k}\left\|x_{k}\right\|_{\mathcal{L}_{\infty}} \leq\left(\frac{\rho}{n}\right) \sum_{k>M} a_{k} \epsilon^{k} \beta^{k} \\
& =\left(\frac{\rho}{n}\right) \text { Remainder }_{M}(h(\beta \epsilon)) .
\end{aligned}
$$

The convergence statement follows by noting that $h(\beta \epsilon)$ can be developed in a convergent Taylor expansion about $\epsilon=0$ in a radius $\beta \epsilon \leq 1+2 \beta-2 \sqrt{\beta+\beta^{2}}$.

Comments: To illustrate the result we compute some terms of the series. Dropping the argument $\tau$

\footnotetext{
${ }^{1} \mathrm{~A}$ second solution is discarded because of incorrect initial conditions.
} 
inside the integral, the first few $x_{k}$ read

$$
\begin{aligned}
x_{2}(t)= & \int_{0}^{t} \mathrm{e}^{A(t-\tau)} f^{[2]}\left(x_{1}, x_{1}\right) d \tau \\
x_{3}(t)= & \int_{0}^{t} \mathrm{e}^{A(t-\tau)}\left\{2 f^{[2]}\left(x_{2}, x_{1}\right)+f^{[3]}\left(x_{1}, x_{1}, x_{1}\right)\right\} d \tau \\
x_{4}(t)= & \int_{0}^{t} \mathrm{e}^{A(t-\tau)}\left\{2 f^{[2]}\left(x_{3}, x_{1}\right)+f^{[2]}\left(x_{2}, x_{2}\right)\right. \\
& \left.\quad+3 f^{[3]}\left(x_{2}, x_{1}, x_{1}\right)+f^{[4]}\left(x_{1}, x_{1}, x_{1}, x_{1}\right)\right\} d \tau .
\end{aligned}
$$

Higher order terms can be easily computed on symbolic manipulation software.

A second remark concerns the truncation error estimate in equation (5). At $M=0$, the estimate turns into an upper bound on the solution $\|x\|_{\mathcal{L}_{\infty}}$. In other words, whenever convergence is guaranteed we have

$$
\|x\|_{\mathcal{L}_{\infty}} \leq\left(\frac{\rho}{n}\right)\left(1-\sqrt{\frac{\beta}{1+\beta}}\right) .
$$

The convergence properties are similar to the ones discussed in [10, Chapter 8]. The definition in quation (4) guarantees that $\beta<\infty$ for any stable system. Hence, the condition

$$
\beta \epsilon \leq 1+2 \beta-2 \sqrt{\beta+\beta^{2}}
$$

implies that for any stable system there exists a small enough $\epsilon^{\star}=\epsilon^{\star}(\beta)$ such that for all $\epsilon<\epsilon^{\star}$ the series converges. Alternatively, given a specific value of $\epsilon$, convergence is assured by finding a small enough $\beta$, that is, by finding a small enough $\left\|\mathrm{e}^{A t}\right\|_{\mathcal{L}_{1}}$. This is always possible since this norm goes to zero as the length of interval $I$ vanishes.

\section{Second order polynomial systems}

Polynomial vector fields are common ${ }^{2}$ in example applications, see [4], and it is instructive to investigate whether simpler expressions or stronger convergence properties might be available for this subclass. Low order polynomial systems are of independent interest in the study of normal forms. Finally, series expansions for mechanical systems $[2,3]$ are related to the case of second order polynomial nonlinearities.

Motivated by this reasoning, we present here simpler expressions with stronger convergence properties for systems described by a vector field $f$ whose components are low order polynomial functions. For simplicity, we consider the case of a control system with only a "quadratic" nonlinearity, i.e., the only nonvanishing

\footnotetext{
${ }^{2}$ Systems may be written in polynomial form via coordinate transformations as well as via dynamic extension.
}

tensors are $f^{[1]}$ and $f^{[2]}$. In equation we mean:

$$
\begin{aligned}
\dot{x}(t, \epsilon) & =A x(t, \epsilon)+f^{[2]}(x(t, \epsilon), x(t, \epsilon))+\epsilon g(t) \\
x(0, \epsilon) & =0 .
\end{aligned}
$$

Proposition 4.1 Consider the initial value problem in equation (8). The solution $x: I \times \mathbb{R}_{+} \mapsto \mathbb{R}^{n}$ satisfies

$$
\begin{aligned}
x(t, \epsilon) & =\sum_{k=1}^{+\infty} \epsilon^{k} x_{k}(t) \\
x_{1}(t) & =\int_{0}^{t} \mathrm{e}^{A(t-\tau)} g(\tau) d \tau \\
x_{k}(t) & =\sum_{i=1}^{k-1} \int_{0}^{t} \mathrm{e}^{A(t-\tau)} f^{[2]}\left(x_{i}(\tau), x_{k-i}(\tau)\right) d \tau, \quad k \geq 2 .
\end{aligned}
$$

Without loss of generality assume $\|g\|_{\mathcal{L}_{\infty}}=\left\|f^{[2]}\right\|_{\mathcal{L}_{\infty}}$, and compute

$$
\beta=2\left\|\mathrm{e}^{A t}\right\|_{\mathcal{L}_{1}}\left\|f^{[2]}\right\|_{\mathcal{L}_{\infty}}
$$

If $\beta^{2} \epsilon<1$, the series converges absolutely and uniformly in $t \in I$, and for all integers $M$ the truncation error is bounded by

$$
\left\|x-\sum_{k=1}^{M} x_{k}\right\|_{\mathcal{L}_{\infty}} \leq \frac{1}{\beta} \operatorname{Remainder}_{M}\left(1-\sqrt{1-\beta^{2} \epsilon}\right) .
$$

Comments: The proof of Proposition 4.1 is straightforward given the statement and proof in the previous section. We refer the interested reader to a later publication. Like in the comments after Proposition 3.1, we present the first few terms of the series. Equation (11) reads:

$$
\begin{aligned}
& x_{2}(t)=\int_{0}^{t} \mathrm{e}^{A(t-\tau)} f^{[2]}\left(x_{1}, x_{1}\right) d \tau \\
& x_{3}(t)=\int_{0}^{t} \mathrm{e}^{A(t-\tau)}\left\{2 f^{[2]}\left(x_{2}, x_{1}\right)\right\} d \tau \\
& x_{4}(t)=\int_{0}^{t} \mathrm{e}^{A(t-\tau)}\left\{2 f^{[2]}\left(x_{3}, x_{1}\right)+f^{[2]}\left(x_{2}, x_{2}\right)\right\} d \tau .
\end{aligned}
$$

Note the agreement with the expressions for the analytic case. The polynomial nature of the control system (8) leads to simplifications in the bound on the solution $\|x\|_{\mathcal{L}_{\infty}}$ and in the computation of the the parameter $\beta$ which roughly describes the nonlinearity and stability of the system. In computing $\beta$, no norms over complex planes are required. Whenever convergence is guaranteed it holds

$$
\|x\|_{\mathcal{L}_{\infty}} \leq \frac{1}{\beta}
$$

Even though the estimates for polynomial vector fields have a simpler expression, they qualitatively agree with 
the ones for the more general analytic vector field case: given the parameter $\beta$, the series converges for all forcing terms smaller in magnitude than a constant $\epsilon^{\star}=\epsilon^{\star}(\beta)$.

\section{Conclusions}

We have presented series expansions for the evolution of a large class of nonlinear control systems. One important feature is the detailed convergence analysis. In particular, assuming the origin is an exponentially stable equilibrium point, we provide sufficient conditions for convergence over the infinite horizon.

A number of research avenues remain open. It is of interest to characterize the relationship with the ChenFliess series, to extend Proposition 3.1 to systems with generic inputs, and to pursue further simplifications via normal form theory. Finally, the simplicity and convergence properties of the novel series might help in areas such as nonlinear controllability, trajectory generation, and numerical optimal control.

\section{References}

[1] R. W. Brockett. Volterra series and geometric control theory. Automatica, 12:167-176, 1976.

[2] F. Bullo. A series describing the evolution of mechanical control systems. In IFAC World Conference, volume E, pages 479-485, Beijing, China, July 1999.

[3] F. Bullo. Series expansions for the evolution of mechanical control systems. SIAM Journal on Control and Optimization, December 1999. Submitted.

[4] F. Bullo, N. Ehrich Leonard, and A. D. Lewis. Controllability and motion algorithms for underactuated Lagrangian systems on Lie groups. IEEE Transactions on Automatic Control, 45(8), 2000.

[5] M. Fliess. Fonctionelles causales non linéares et indeterminées non commutatives. Bull. Soc. Math., 109:3-40, 1981.

[6] E. G. Gilbert. Functional expansions for the response of nonlinear differential systems. IEEE Transactions on Automatic Control, 22(6):909$921,1977$.

[7] A. Isidori. Nonlinear Control Systems. Springer Verlag, New York, NY, third edition, 1995. ISBN 3540199160 .

[8] M. Kawski. High-order small-time local controllability. In H. J. Sussmann, editor, Nonlinear Controllability and Optimal Control, pages 441-477. Dekker, 1990. ISBN 0824782585.
[9] M. Kawski and H. J. Sussmann. Noncommutative power series and formal Lie-algebraic techniques in nonlinear control theory. In U. Helmke, D. Pratzel-Wolters, and E. Zerz, editors, Operators, Systems, and Linear Algebra, pages 111-128. Teubner, Stuttgart, Germany, 1997.

[10] H. K. Khalil. Nonlinear Systems. Prentice Hall, Englewood Cliffs, NJ, second edition, 1995. ISBN 0132280248 .

[11] D. E. Knuth. The Art of Computer Programming, Volume 1, Fundamental Algorithms. Addison Wesley, Reading, MA, third edition, 1997. ISBN 0201896834.

[12] S. G. Krantz. Function Theory of Several Complex Variables. Pure and Applied Mathematics. John Wiley and Sons, New York, NY, 1982. ISBN 0471093246.

[13] G. Lafferriere and H. J. Sussmann. A differential geometric approach to motion planning. In Z. Li and J. F. Canny, editors, Nonholonomic Motion Planning, pages 235-270. Kluwer Academic Publishers, 1993. ISBN 0792392752.

[14] N. Ehrich Leonard and P. S. Krishnaprasad. Motion control of drift-free, left-invariant systems on Lie groups. IEEE Transactions on Automatic Control, 40(9):1539-1554, 1995.

[15] C. Lesiak and A. J. Krener. The existence and uniqueness of Volterra series for nonlinear systems. IEEE Transactions on Automatic Control, 23(6): 1090-1095, 1978.

[16] R. I. McLachlan, G. R. W. Quispel, and N. Robidoux. Unified approach to Hamiltonian systems, Poisson systems, gradient systems, and systems with Lyapunov functions or first integrals. Physical Review Letters, 81(12):2399-403, 1998.

[17] W. J. Rugh. Nonlinear System Theory: The Volterra/Wiener Approach. John Hopkins University Press, Baltimore, Maryland, 1981. ISBN 0801825490 .

[18] J. A. Sanders and F. Verhulst. Averaging Methods in Nonlinear Dynamical Systems. Springer Verlag, New York, NY, 1985. ISBN 0387962298.

[19] H. J. Sussmann. A product expansion of the Chen series. In C. I. Byrnes and A. Lindquist, editors, Theory and Applications of Nonlinear Control Systems, pages 323-335. Elsevier, Oxford, UK, 1986. ISBN 0444700552.

[20] H. J. Sussmann. A general theorem on local controllability. SIAM Journal on Control and Optimization, 25(1):158-194, 1987. 